Cite this: J. Mater. Chem. A, 2014, 2 , 6218

Received 9th December 2013 Accepted 28th January 2014

DOI: $10.1039 / \mathrm{c} 3 \operatorname{ta} 15112 \mathrm{C}$

www.rsc.org/MaterialsA

\section{The influence of microstructure on charge separation dynamics in organic bulk heterojunction materials for solar cell applications}

\author{
M. Scarongella, ${ }^{a}$ A. A. Paraecattil, ${ }^{a}$ E. Buchaca-Domingo, ${ }^{b}$ J. D. Douglas, ${ }^{c}$ S. Beaupré, ${ }^{d}$ \\ T. McCarthy-Ward, ${ }^{e}$ M. Heeney, ${ }^{\text {e }}$ J.-E. Moser, ${ }^{a}$ M. Leclerc, ${ }^{d}$ J. M. J. Fréchet, ${ }^{c f}$ \\ N. Stingelin ${ }^{\mathrm{b}}$ and N. Banerji ${ }^{\star a}$
}

Light-induced charge formation is essential for the generation of photocurrent in organic solar cells. In order to gain a better understanding of this complex process, we have investigated the femtosecond dynamics of charge separation upon selective excitation of either the fullerene or the polymer in different bulk heterojunction blends with well-characterized microstructure. Blends of the PBTTT and PBDTTPD polymers with PCBM gave us access to three different scenarios: either a single intermixed phase, an intermixed phase with additional pure PCBM clusters, or a three-phase microstructure of pure polymer aggregates, pure fullerene clusters and intermixed regions. We found that ultrafast charge separation (by electron or hole transfer) occurs predominantly in intermixed regions, while charges are generated more slowly from excitons in pure domains that require diffusion to a charge generation site. The pure domains are helpful to prevent geminate charge recombination, but they must be sufficiently small not to become exciton traps. By varying the polymer packing, backbone planarity and chain length, we have shown that exciton diffusion out of small polymer aggregates in the highly efficient PBDTTPD:PCBM blend occurs within the same chain and is helped by delocalization.

\section{Introduction}

Encouraging power conversion efficiencies approaching 10\% have been reported for organic solar cells. ${ }^{\mathbf{1 - 4}}$ Much remains nevertheless to be learned about how light is converted to charges and subsequent current in those devices. Typically, an electrondonating conjugated polymer is blended with an electronaccepting fullerene in the photovoltaic active layer, spontaneously forming an interpenetrating network known as the bulk heterojunction $(\mathrm{BHJ}) .^{5-7}$ According to the most widespread picture of charge generation, the $\mathrm{BHJ}$ consists of 10-20 nm pure polymer and fullerene domains. Upon light absorption, an exciton is formed in the polymer phase and diffuses to a fullerene interface, where charge separation (CS) takes place via electron transfer (ET)

anstitute of Chemical Sciences \& Engineering, Ecole Polytechnique Fédérale de Lausanne (EPFL), SB ISIC GR-MO, Station 6, CH-1015 Lausanne, Switzerland. E-mail: natalie.banerji@epfl.ch

${ }^{b}$ Centre for Plastic Electronics and Department of Materials, Imperial College London, Exhibition Road, London, SW7 2AZ, UK

${ }^{c}$ Department of Chemistry, University of California, 727 Latimer Hall, Berkeley, California 94720-1460, USA

${ }^{d}$ Department of Chemistry, Université Laval, G1K 7P4 Quebec, Quebec, Canada ${ }^{e}$ Centre for Plastic Electronics and Department of Chemistry, Imperial College London, Exhibition Road, London, SW7 2AZ, UK

${ }^{f}$ King Abdullah University of Science and Technology (KAUST), Thuwal, 23955-6900, Saudi Arabia to the acceptor. The electron and hole are then transported to opposite electrodes along segregated acceptor and donor pathways. This simple picture suffers from severe limitations. The long-range exciton diffusion is for example inconsistent with frequently reported ultrafast appearance of charges within 100 fs. ${ }^{8-10}$ Also, CS via hole transfer (HT) from photoexcited fullerene is not accounted for. ${ }^{11-18}$ Moreover, the microstructure of the $\mathrm{BHJ}$ is much more complex in most polymer:fullerene blends. Amorphous and/or crystalline pure domains often coexist with a phase where the polymer and fullerene are intimately mixed. ${ }^{19-23}$ This has strong implications on the photophysics of the materials. ${ }^{24-30}$

In the present contribution, we demonstrate that the dynamics of charge generation in polymer:fullerene blends is largely determined by the microstructure. To that aim, we have used femtosecond transient absorption (TA) spectroscopy to investigate CS in several systems with well-characterized $\mathrm{BHJ}$ structure. By selectively exciting either the polymer or fullerene, we have targeted either electron or hole transfer and evaluated the effect of having pure donor or acceptor domains and intermixed regions on both CS pathways. The first set of samples consists of blends of pBTTT (poly(2,5-bis(3-hexadecyl-thiophen-2yl)thieno [3,2- $b]$ thiophene $)$ ) with various fractions of PCBM $([6,6]-$ phenyl $\mathrm{C}_{60}$ butyric acid methyl ester). The pBTTT polymer is known for its excellent charge mobility in field effect transistor devices. ${ }^{31-33}$ Even if they yield moderate efficiency in solar cells, pBTTT:fullerene blends are particularly suitable model systems to 
study microstructural effects. ${ }^{22}$ Indeed, it has been shown that the fullerene molecules intercalate between the side-chains of ordered polymer domains, forming a well-defined co-crystalline phase, while excess fullerenes aggregate into relatively phase-pure clusters. ${ }^{19,22,23,26}$ The second investigated polymer is PBDTTPD (poly(benzo[1,2- $\left.b: 4,5-b^{\prime}\right]$ dithiophene-alt-thieno[3,4-c]pyrrole-4,6dione), which is one of the highest performing organic solar cell materials with up to $8.5 \%$ power conversion efficiency when blended with fullerenes. ${ }^{2,24,34-36}$ In contrast to the pBTTT systems with either a single co-crystalline phase or a two-phase scenario of co-crystals and pure fullerene domains, PBDTTPD:PCBM gives us access to an essentially three-phase microstructure consisting of pure polymer aggregates, pure fullerene domains and molecularly intermixed polymer:fullerene regions. ${ }^{24}$ We have investigated the effect of varying the polymer molecular weight and of slightly tuning the polymer packing by side-chain variation. ${ }^{2,35}$ Overall, the results presented here point to a strong correlation between the $\mathrm{BHJ}$ microstructure and the CS dynamics. Ultrafast CS occurs predominantly in intermixed regions, while charges are generated more slowly from excitons in pure domains that require diffusion to a charge generation site. The pure domains are necessary to prevent geminate charge recombination (gCR), but they must be small and adapted to efficient exciton diffusion in terms of delocalization and polymer chain length.

\section{Experimental part}

\section{Samples}

The pBTTT polymer $\left(M_{\mathrm{n}}=34 \mathrm{kDa} ; M_{\mathrm{w}}=66 \mathrm{kDa}\right.$, molecular structure in Fig. 1A) was synthesised as previously reported, ${ }^{33}$ while PCBM for the corresponding blends was purchased from Solenne and used without further purification. Solutions of a pBTTT and PCBM mixture in $1: 1$ and $1: 4$ weight ratio were prepared in 1,2-ortho-dichlorobenzene (1,2-o-DCB, Aldrich). The total concentration of the pBTTT:PCBM mixtures was $20 \mathrm{mg}$ $\mathrm{mL}^{-1}$ and $50 \mathrm{mg} \mathrm{mL}^{-1}$, respectively. All solutions were left stirring for more than 4 hours at $100{ }^{\circ} \mathrm{C}$ to fully dissolve the active material. Films were then deposited on glass by wire-bar coating from hot solutions $\left(\sim 85-90{ }^{\circ} \mathrm{C}\right)$ at $35{ }^{\circ} \mathrm{C}$ to ensure the formation of the co-crystals. Prior to measurements, oxygen was removed by keeping them for 24 hours in a vacuum. The samples were then sealed in an inert measuring chamber within a glovebox. The film thickness was approximately 100-110 nm with a maximum absorption (optical density) in the visible range of about 0.5 , as measured with a PerkinElmer Lambda 950 spectrophotometer.

The different PBDTTPD batches were synthesised according to previously published procedures..$^{35,36}$ Various side-chains were attached to the polymer, yielding structural derivatives designated as P1, P2 and P3 (molecular structures in Fig. 1B). Additionally, we investigated different molecular weight fractions: P1 with $M_{\mathrm{n}}=6 \mathrm{kDa}(\mathrm{PDI}=2.6)$ and $M_{\mathrm{n}}=28 \mathrm{kDa}(\mathrm{PDI}=$ 1.6); P3 with $M_{\mathrm{n}}=27 \mathrm{kDa}(\mathrm{PDI}=1.9)$ and $M_{\mathrm{n}}=36 \mathrm{kDa}(\mathrm{PDI}=$ 1.9). For the blends, PCBM (99\%) was purchased from Ossila Ltd. Films were prepared according to the method yielding the highest solar cell efficiency for the given batch. For the wavelength-dependent studies with P3 $\left(M_{\mathrm{n}}=14.7 \mathrm{kDa}\right.$, PDI $\left.=2.15\right)$, the neat polymer as well as the PBDTTPD:PCBM mixture $1: 2$ weight ratio) were dissolved in anhydrous 1,2-o-DCB at a $10 \mathrm{mg}$ $\mathrm{mL}^{-1}$ polymer concentration inside a glovebox. They were

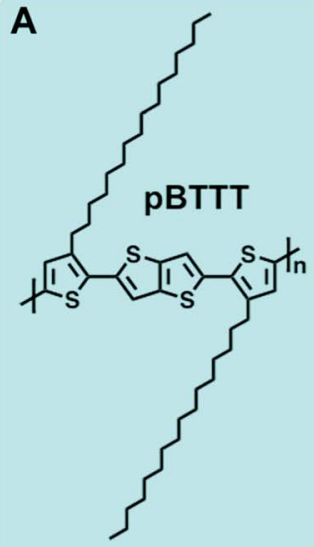

pBTTT:PCBM (1:1)

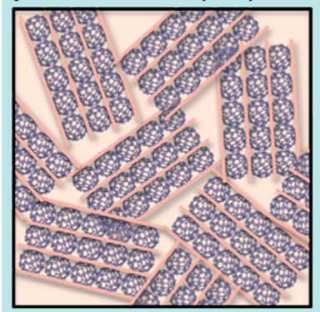

B
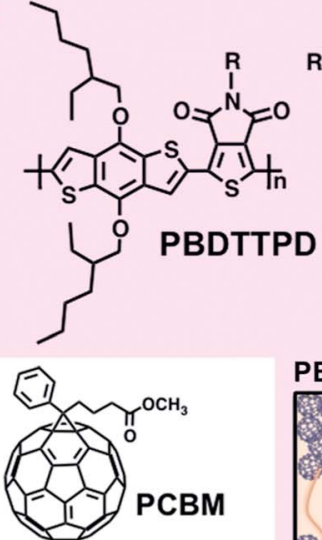

pBTTT:РCBM (1:4)

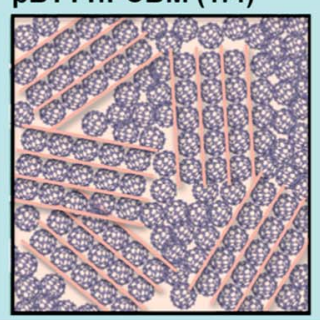

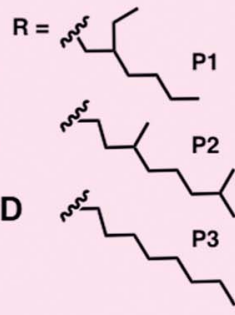

PBDTTPD:PCBM

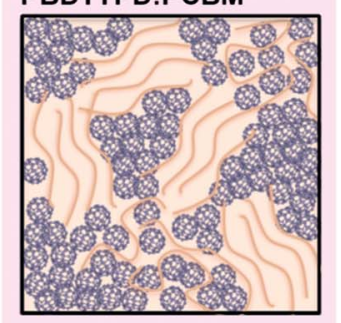

$=$ PCBM

$=$ Polymer Chains
C

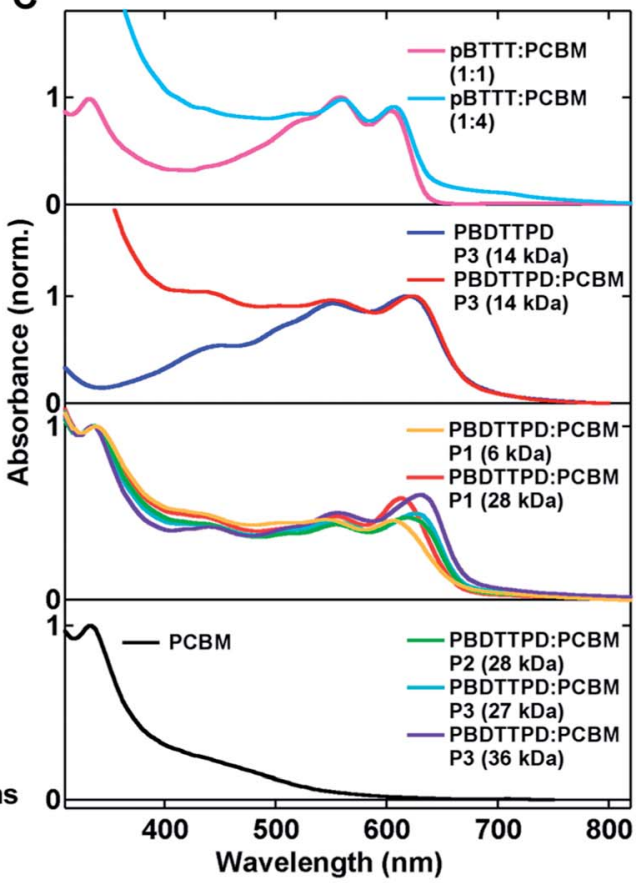

Fig. 1 (A and B) Molecular structure of the investigated molecules and schematic representation of the polymer:fullerene bulk heterojunction microstructure. (C) Normalized steady-state absorption spectra of the investigated thin films. 
stirred for 48 hours at $100-120{ }^{\circ} \mathrm{C}$. The hot solutions were spincoated at $1000 \mathrm{rpm}$ for 2 minutes onto glass substrates that had previously been sonicated in water/acetone/isopropanol and plasma cleaned. For the comparison of the P1, P2 and P3 blends of similar molecular weight $\left(M_{\mathrm{n}}=27-28 \mathrm{kDa}\right.$, PDI $\left.=1.6-1.9\right)$, a $1: 1.5$ by weight polymer:fullerene mixture $\left(10 \mathrm{mg} \mathrm{mL}^{-1}\right.$ polymer content) was dissolved overnight in anhydrous chlorobenzene (CB) at $115^{\circ}$ and then spin coated at $2500 \mathrm{rpm}$. The same procedure was used for the comparison of the different molecular weights with P1 and P3. The film thickness was in all cases around $100 \mathrm{~nm}$, with a maximum visible absorbance about 0.3-0.4. Again, measurements were carried out in an inert sample chamber. Although microstructural degradation has been reported for PBDTTPD:PCBM (in particular for the high molecular weight samples), ${ }^{37}$ we did not notice any effect on the TA measurement recorded immediately, one week and several weeks after film preparation, indicating that any degradation insignificantly influenced the investigated properties.

\section{Transient absorption spectroscopy}

The TA spectra were recorded using femtosecond pulsed laser pump-probe spectroscopy. Excitation at $530 \mathrm{~nm}$ and $540 \mathrm{~nm}$ was used to excite the polymer in the PBDTTPD and pBTTT blends, respectively. For those wavelengths, the pump beam was generated with a commercial two-stage non-collinear optical parametric amplifier (NOPA-Clark, MXR) from the $778 \mathrm{~nm}$ output of a Ti:sapphire laser system with a regenerative amplifier providing femtosecond pulses at a repetition rate of $1 \mathrm{kHz}$. Compression of the NOPA output with two prisms leads to 50-60 fs pulse duration. Alternatively, the samples were excited at $390 \mathrm{~nm}$ (where there is significant PCBM absorption), after frequency doubling the fundamental $778 \mathrm{~nm}$ beam in a BIBO crystal (about $150 \mathrm{fs}$ pulse duration). The pulse energy at the sample was adjusted within the tens of nano-joule range and the beam diameter was around $1 \mathrm{~mm}$ (determined precisely with a BC106-Vis Thorlabs beam profiler, $1 / e^{2}$ cut-off). For each measurement, identical dynamics at slightly higher and lower power assured complete absence of any intensity-induced bimolecular effects. To be in this regime required a fluence of $6-8 \mu \mathrm{J} \mathrm{cm}^{-2}$ for the pBTTT samples. Taking into account the absorbance at the excitation wavelength and the photon energy, this corresponds to a flux of absorbed photons of $1-2 \times 10^{13}$ photons per $\mathrm{cm}^{2}$. A lower fluence of $2-4 \mu \mathrm{J} \mathrm{cm} \mathrm{cm}^{-2}\left(2-6 \times 10^{12}\right.$ photons per $\left.\mathrm{cm}^{2}\right)$ was necessary for the PBDTTPD blends. For the comparison of the two excitation wavelengths using the P3:PCBM sample, particular care was taken to keep the photon flux constant at $5.9 \times 10^{12}$ photons per $\mathrm{cm}^{2}$. The low pump powers used in the experiments also prevented any sample degradation, as confirmed by excellent reproducibility of the data.

The probe beam for the TA measurements consisted of a white light continuum (350-1000 $\mathrm{nm})$, generated by passing a portion of the $778 \mathrm{~nm}$ amplified Ti:sapphire output through a 5 $\mathrm{mm}$ thick $\mathrm{CaF}_{2}$ plate (in constant motion to avoid burning). Either a $750 \mathrm{~nm}$ low pass or an $850 \mathrm{~nm}$ high pass filter was used to remove the remaining fundamental intensity from the white light. The visible and n-IR parts of the spectrum were thus recorded separately (and sometimes needed to be slightly scaled). The probe intensity was always less than the pump intensity $(<10 \mathrm{~nJ})$ and the spot size was much smaller (about $0.2 \mathrm{~mm}$ ). The probe pulses were time delayed with respect to the pump pulses using a computerized translation stage. The probe beam was split before the sample into a signal beam (transmitted through the sample and crossed with the pump) and a reference beam. The signal and reference were detected with a pair of $163 \mathrm{~mm}$ spectrographs (Andor Technology, SR163) equipped with a $512 \times 58$ pixels back-thinned CCD (Hamamatsu S07030-0906) and assembled by Entwicklungsbüro Stresing, Berlin. To improve sensitivity, the pump light was chopped at half the amplifier frequency, and the transmitted signal intensity was recorded shot by shot. It was corrected for intensity fluctuations using the reference beam. The transient spectra were averaged until the desired signal-to-noise ratio was achieved (5000-6000 times). The polarization of the probe pulses was at a magic angle relative to that of the pump. All spectra were corrected for the chirp of the white-light probe pulses.

\section{Analysis of the transient absorption dynamics}

Dynamics corresponding to different probe wavelengths were obtained by taking cross-sections through the chirp-corrected TA spectra recorded at about 350 different time delays in the -1 ps to $1 \mathrm{~ns}$ range. The interpretation of such dynamics is not trivial, given the complexity of polymer:fullerene blends. First, there are often overlapping broad spectral signatures, making assignment of the dynamics at a particular probe wavelength to a unique species or process difficult. Then, the time evolution of the TA signals can be influenced by multiple processes, for example the pure charge generation dynamics might be entangled with spectral shifts due to relaxation of the neutral and charged species. Moreover, CS can be strongly multiphasic due to different distances and orientations between the donor and the acceptor. This is further complicated if it is preceded by exciton diffusion over a variety of distances. For all the above reasons, the analysis of a single-wavelength TA dynamics is typically not very meaningful. Instead, we have opted for global analysis, i.e. for simultaneous analysis of the TA dynamics taken every $5 \mathrm{~nm}$ throughout the spectrum. ${ }^{38}$ Apart from being more robust to noise-related artefacts encountered at a single wavelength, this method allows for correlating the evolution of different signatures in the TA spectrum, greatly facilitating the interpretation.

Given the complexity of the TA dynamics described above, it is very challenging to devise a kinetic model that accurately and comprehensively describes all the dynamics. The number of parameters would be so high that even an inappropriate model could very well reproduce the experimental data. Therefore, we have chosen not to impose any specific model on our results, but instead to globally fit multiexponential functions to the dynamics. An analysis using the sum of exponential functions is meaningful when there are several independent exponential processes occurring on well-separated time scales. When a process is itself multiphasic (for example because it is limited by diffusion over a variety of distances), this also leads to 
multiexponentiality. Thus, a Gaussian distribution of time constants for highly multiphasic processes can crystallize into only a few average exponential time constants. ${ }^{39}$ An analysis with an appropriate model is in this case physically more meaningful. Nevertheless, the multiexponential analysis perfectly suits the purpose for the level of discussion required here. It allows evaluating whether a process is multiphasic and estimating the time scale on which it occurs. Moreover, the sign of the pre-exponential factor indicates whether the process associated with the time constant leads to a rise or a decay of the TA signal at a given probe wavelength. By plotting the preexponential factors as a function of probe wavelength (yielding amplitude spectra), we can identify at one glimpse concerted changes in different parts of the transient spectrum. This global appreciation of the evolution of several bands consolidates the assignment of the observed dynamics to physical processes. The shape of the amplitude spectra also allows disentangling different processes if they occur on a similar time scale and appear mixed into the same exponential time constant.

\section{Results and discussion}

\section{Part I: charge generation in pBTTT:PCBM}

The steady-state absorption spectra of pBTTT:PCBM thin films prepared with a $1: 1$ and $1: 4$ (by weight) polymer:fullerene ratio are shown in the first panel of Fig. 1C. The polymer has a predominant absorption band between 450 and $650 \mathrm{~nm}$. PCBM absorbs mostly at lower wavelengths $(<500 \mathrm{~nm})$, explaining the enhanced relative absorption in this region for the $1: 4$ blend. The observed vibronic structure in the polymer band is typical if the latter is intimately intermixed with fullerenes. ${ }^{28}$ Indeed, complete intercalation of the PCBM molecules between the side chains of pBTTT has previously been demonstrated for the $1: 1$ blend ${ }^{26}$ For this sample, we therefore have a single co-crystalline phase, where all polymer and fullerene molecules are in close proximity to each other, as schematically depicted in Fig. 1A. For the sample with higher PCBM content (1: 4 blend), fractions of the fullerene still form a co-crystal with the polymer, but the excess PCBM leads to the development of relatively phase-pure aggregated PCBM domains. ${ }^{26}$ Those pure fullerene regions have been identified to be necessary for photocurrent generation in solar cells, the efficiency being very low if only the co-crystals are present. ${ }^{22}$

Selected TA spectra of the $1: 1$ and $1: 4$ blends of pBTTT:PCBM are shown for two excitation wavelengths $(390 \mathrm{~nm}$ and $540 \mathrm{~nm}$ ) in Fig. 2. At $540 \mathrm{~nm}$, predominantly the polymer is excited, while mainly PCBM absorbs at $390 \mathrm{~nm}$ with a weak contribution from pBTTT (Fig. 1C). Let us start by considering the $1: 1$ sample. At first glance, there is no striking difference between the TA spectra and their evolution recorded at the two excitation wavelengths. The spectra are dominated by a structured negative band in the $480-620 \mathrm{~nm}$ region, which coincides with the steady-state absorption spectrum of pBTTT and is therefore assigned to the ground state bleaching (GSB) of the polymer. The presence of the GSB indicates that pBTTT is in a state other than the ground state (e.g. excited, charged, triplet...). There is also a broad positive band extending from 625-1000 nm. This band has a pronounced peak around $630 \mathrm{~nm}$ (partially overlapping with the GSB) and slightly rises towards the longer wavelengths. We assign this broad band to

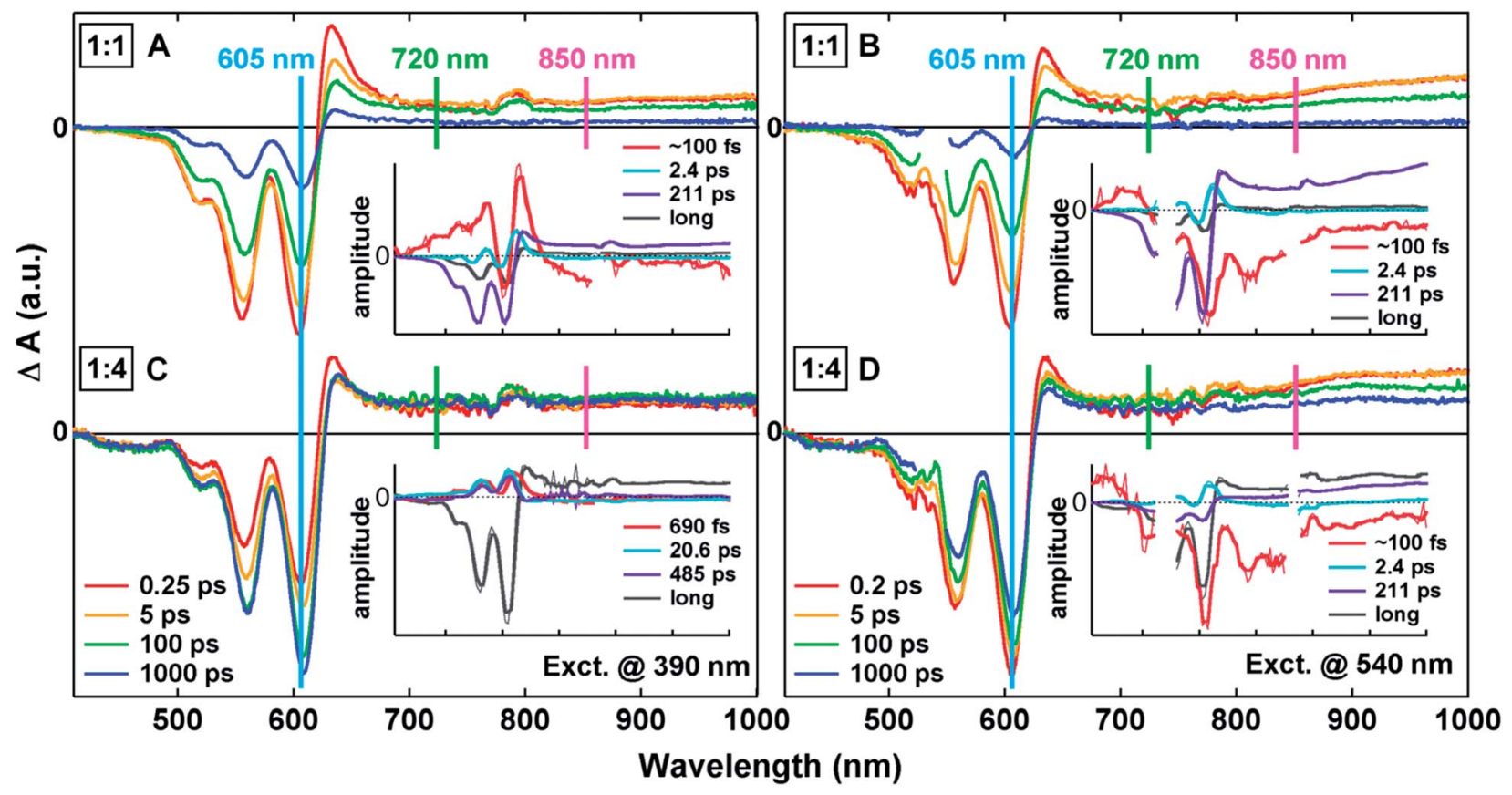

Fig. 2 Transient absorption spectra recorded at selected time delays for pBTTT:PCBM (A) $1: 1$ blend following $390 \mathrm{~nm}$ excitation, (B) $1: 1$ blend following $540 \mathrm{~nm}$ excitation, (C) 1 : 4 blend following $390 \mathrm{~nm}$ excitation, and (D) $1: 4$ blend following $540 \mathrm{~nm}$ excitation. Noise around $780 \mathrm{~nm}$ is caused by remaining fundamental laser light in the probe beam. The insets show the amplitude spectra associated with the time constants obtained by multiexponential global analysis of the data (the thicker solid lines are smoothed, as guide to the eye). 
absorption from positive charges (polarons) on pBTTT, in good agreement with reported photoinduced absorption (PIA) and charge modulation spectroscopy (CMS) measurements. ${ }^{40,41}$ There is little change to the shape of the TA spectra when going from the earliest timedelay to the longest (from 200 fs to $1 \mathrm{~ns}$ ), and the $630 \mathrm{~nm}$ peak is immediately present. This indicates that the majority of charges are formed shortly after excitation at either wavelength.

The occurrence of ultrafast CS in the $1: 1$ blend is in agreement with the observed TA dynamics shown in Fig. 3 for selected probe wavelengths in the GSB $(605 \mathrm{~nm})$ and charge absorption $(720 \mathrm{~nm}, 840 \mathrm{~nm})$. With $540 \mathrm{~nm}$ excitation, where our time resolution was as short as 50-60 fs, an ultrafast $(<300$ fs) component pointing to a rise of charge absorption is visible. We are confident that it is real, since we carefully distinguished it from the signal rise within the laser pulse and verified that there is no interference by artefacts around timedelay zero. The global analysis confirms that the ultrafast process is observed throughout the TA spectrum and is associated with a time constant of $\sim 100 \mathrm{fs}$. The corresponding amplitude spectrum is shown in the inset of Fig. $2 \mathrm{~B}$ in red and further supports the assignment to ultrafast ET from excited pBTTT. Indeed, ET should lead to a disappearance of TA signatures related to the polymer exciton (stimulated emission (SE) and excited state absorption (ESA)), with concomitant rise of charge absorption. In the amplitude spectrum, the structured negative band to the red side of the GSB, which almost mirrors the latter, is highly

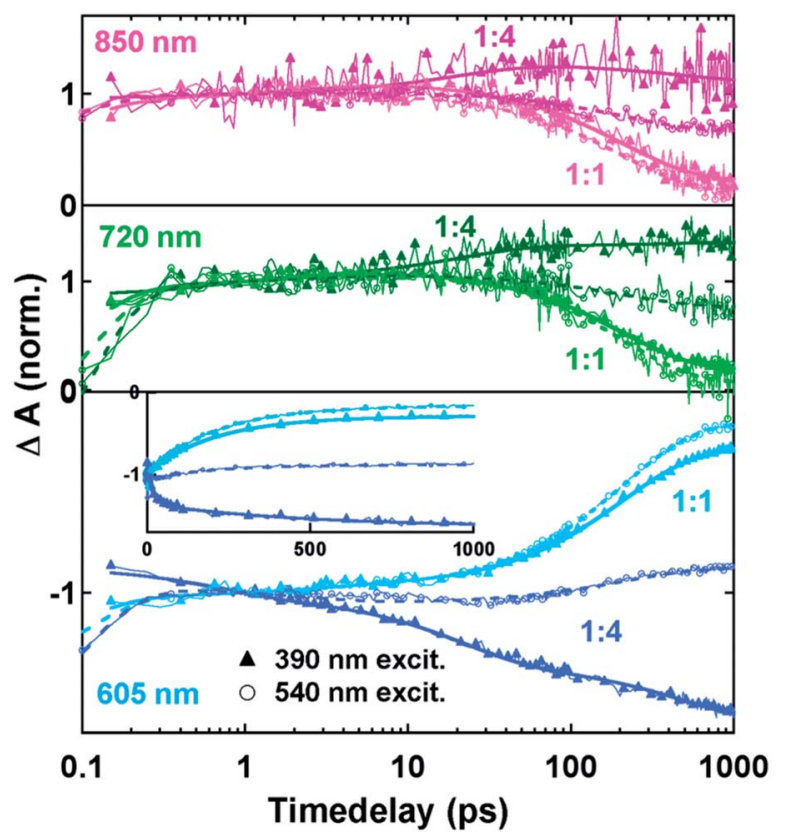

Fig. 3 TA dynamics at selected probe wavelengths $(605 \mathrm{~nm}, 720 \mathrm{~nm}$ and $850 \mathrm{~nm}$ ) recorded for the $1: 1$ and $1: 4$ blends (slightly lighter and darker colour, respectively) of $\mathrm{pBTTT:PCBM}$ following excitation at $390 \mathrm{~nm}$ (triangles) or $540 \mathrm{~nm}$ (circles). The time profiles were normalized at 1 ps to facilitate comparison. The solid lines represent the best multiexponential fit obtained by global analysis (solid lines for $390 \mathrm{~nm}$ excitation, dashed lines for $540 \mathrm{~nm}$ excitation). Dynamics are shown on a logarithmic time axis, except in the inset. indicative of decay of the polymer's SE. In the corresponding $200 \mathrm{fs}$ TA spectrum, the indent in the $670-800 \mathrm{~nm}$ region is possibly the remaining tail of this SE. In the amplitude spectrum, the pre-exponential factor related to the $\sim 100$ fs process is negative all the way up to $1000 \mathrm{ps}$, suggesting a rise of the charge absorption band, which partly overlaps with the SE. Moreover, the amplitude is positive in the $420-500 \mathrm{~nm}$ region, pointing to the growth of a negative band, which we assign to the GSB of PCBM based on the coincidence with the steady-state absorption spectrum. ET is expected to lead to a rise of this GSB of PCBM, since the latter is not directly excited, but removed from the ground state via the CS process.

Let us now turn to what happens with $390 \mathrm{~nm}$ excitation in the $1: 1$ blend. Although it is mainly the PCBM which absorbs at this wavelength, the early $250 \mathrm{fs}$ TA spectrum shown in Fig. $2 \mathrm{~A}$ is dominated by the GSB of the polymer and by charge absorption. This suggests that ultrafast CS has already occurred, this time mainly by HT from photoexcited fullerene, involving the HOMO levels of the donor and acceptor. It has been previously established that HT in polymer:fullerene blends can be as fast as 30 fs. ${ }^{16}$ Given the lower time resolution that we have at $390 \mathrm{~nm}(150 \mathrm{fs})$, it is difficult to resolve the process. Still we could identify the tail of the ultrafast dynamics at the earliest time delays and analyse them roughly using a $\sim 100$ fs time constant. The predominantly positive sign in the 430-650 nm region of the corresponding amplitude spectrum points to an increase of the polymer's GSB, as expected during HT (inset of Fig. 2A). The negative amplitude at higher wavelengths is representative of rise of the charge absorption and may include some decay of the SE of PBTTT during ET, since the polymer is also slightly excited at $390 \mathrm{~nm}$. We relate the ultrafast $(\sim 100 \mathrm{fs})$ charge formation by ET and $\mathrm{HT}$ in the $1: 1$ pBTTT:PCBM blend at both excitation wavelengths to a static quenching process (no exciton diffusion), given the close proximity of the donor and acceptor in the intercalated cocrystals. This is in excellent agreement with reported steadystate and time-resolved emission quenching studies, which have revealed highly efficient (>99\%) quenching of the polymer fluorescence in the $1: 1$ blend. ${ }^{26,28}$ There has been a lot of recent discussion that ultrafast CS in polymer:fullerene blends might compete with relaxation and occur from hot, highly delocalized states. ${ }^{\mathbf{8 , 9 , 4 2 , 4 3}}$ Our results are not in contradiction with such a mechanism, but further discussion is beyond the scope of the present manuscript.

Careful inspection of the TA spectra of the $1: 1$ blend in Fig. $2 \mathrm{~A}$ and $\mathrm{B}$ reveals a slight (about $4 \mathrm{~nm}$ ) red shift of the peaks related to the polymer GSB. This shift leads to dynamics that we could reproduce globally with a 2.4 ps time constant. The corresponding amplitude spectrum (cyan in the insets of Fig. 2) is present at both excitation wavelengths. Its oscillatory shape is characteristic of the alternating rising and decaying dynamics associated with a shift. A red shift of the polymer GSB in a fullerene blend has been previously reported and explained by migration of charges to lower energy sites within an inhomogeneous density of states. ${ }^{44}$ This process is however too slow to account for what we are observing here. Therefore, we tentatively ascribe the shift of the GSB to changes in the pBTTT 
absorption spectrum that occur because of the electric field generated by the presence of charges (Stark effect). ${ }^{41,45}$ The strong similarity of the amplitude spectrum with the first derivative of the steady-state absorption spectrum further supports the involvement of a quadratic Stark effect. The observation of the effect on the 2.4 ps time scale might be caused by some slightly delayed CS (as suggested by the concomitant weak rise of the 690-100 $\mathrm{nm}$ absorption band) and/or by relaxation of the charges. We note that the red shift of the polymer's GSB into the overlapping positive $630 \mathrm{~nm}$ band also occurs during the ultrafast CS processes. This causes for example the oscillatory shape around $615 \mathrm{~nm}$ in the amplitude spectrum of the $\sim 100$ fs time constant with $390 \mathrm{~nm}$ excitation (inset of Fig. 2A). We will address this Stark effect in more detail in a future study involving more specialized techniques.

The evolution of the charges in the $1: 1$ blend at longer times can be followed by considering the dynamics in Fig. 3 . There is decay on the hundreds of picosecond time scale of both the charge absorption $(720 \mathrm{~nm}, 850 \mathrm{~nm})$ and the polymer GSB (605 $\mathrm{nm}$ ), pointing to recombination. Only a small fraction of the charges survive beyond the 1 ns time window, as shown by the weak long-lived plateau in the dynamics. The dynamics with $390 \mathrm{~nm}$ and $540 \mathrm{~nm}$ excitation are quite similar (they were normalized at $1 \mathrm{ps}$ for better visualization), showing that the fate of charges generated by ET and HT is comparable. By global analysis, we estimate in both cases an average time scale of 211 ps for the recombination, with the associated amplitude spectrum essentially showing the decay of the GSB and charge signatures (purple curve, insets of Fig. 2). The much weaker grey amplitude spectrum of similar shape represents the plateau due to the population of long-lived charges. The observed recombination is geminate ( $\mathrm{gCR}$ ), since it is independent of pump intensity. This recombination results from electron-hole pairs that have dissociated within the co-crystalline phase (where the fullerene and polymer are molecularly mixed and hence, in close proximity), but cannot overcome the Coulomb force still binding them together in a charge transfer (CT) state.

Ultrafast formation of free charge carriers via hot CT states, as opposed to dissociation of the relaxed CT state, has been intensively discussed in the literature..$^{4,46-48}$ In the present study, we cannot distinguish between the two mechanisms. But we can say that the intercalated polymer:fullerene microstructure favours gCR, leading to a low yield of free charges. Finally, we note the apparent higher remaining polymer GSB signature with $390 \mathrm{~nm}$ excitation at $1 \mathrm{~ns}$, leading to the small differences in the dynamics recorded at the two pump wavelengths (Fig. 2 and 3). Two reasons could account for this observation. First, it has been shown that the yield of free charges, and thus the fraction of bound charges undergoing gCR, can depend on the generation pathway by ET or HT if the driving force for the two processes is very different. ${ }^{11}$ A larger driving force for HT in pBTTT:PCBM might therefore lead to a slightly weaker contribution of gCR. Second, we cannot exclude the occurrence of some delayed HT components at $390 \mathrm{~nm}$, causing a rise of the GSB and charge signals that partly counterbalances the decay of those features due to gCR. The decay of the signatures related to the fullerene exciton is not expected to be useful to identify delayed HT, since their amplitude is weak and mostly drowned by the intense polymer bands. ${ }^{\mathbf{4 9 , 5 0}}$

The TA spectra of the 1:4 pBTTT:PCBM blend upon $540 \mathrm{~nm}$ excitation of predominantly the polymer are shown in Fig. 2D. The shape is very similar to the one recorded in the $1: 1$ blend (except for a more pronounced fullerene GSB around $450 \mathrm{~nm}$ due to high PCBM loading). The early appearance of the charge signatures points again to ultrafast ET. Indeed a short (hundreds of femtoseconds) component is visible in the dynamics of the 1:4 blend (Fig. 3) and could again be globally reproduced with a $\sim 100$ fs time constant. The amplitude spectrum is very similar to the corresponding one in the $1: 1$ sample, characteristic of ET (inset of Fig. 2D). The slower $2.4 \mathrm{ps}$ process (probably related to a Stark effect), leading to a red shift of the polymer GSB and an oscillatory amplitude spectrum, is again present in the $1: 4$ sample. Overall, the results show that ET is very efficient and fast in both the $1: 1$ and $1: 4$ blends, because the pBTTT chains are in both cases in close proximity to the intercalated PCBM molecules. This agrees with equally efficient $>99 \%$ polymer emission quenching in the $1: 1$ and $1: 4$ blends. $^{26}$ Differences only appear at longer time scales. From the TA spectra and the dynamics, it is immediately clear that the loss of charges due to gCR is significantly reduced in the $1: 4$ blend. This is further confirmed by the decreased weight of the amplitude spectrum associated with the 211 ps gCR component (in purple), and the enhanced weight of the long-lived charges (in grey). The presence of the pure fullerene clusters thus increases the yield of long-lived free charge carriers. This is in excellent agreement with higher solar cell efficiency in the $1: 4$ blend, ${ }^{22}$ and with previous TA, PIA and microwave conductivity results, mostly recorded at much longer times than the femtosecond results presented here..$^{\mathbf{2 6 , 2 8 , 3 0 , 4 0}}$ The high electron mobility in the pure fullerene domains and increased electron affinity compared to intercalated PCBM provide a driving force for the spatial separation of charges formed by exciton dissociation in the co-crystals. ${ }^{26,28}$

Finally, we will consider what happens in the $1: 4$ blend with $390 \mathrm{~nm}$ excitation (Fig. 2C). Given the high PCBM content of the sample, mainly fullerenes (both in the co-crystals and relatively pure clusters) are excited. The early 250 fs TA spectrum shows the polymer's GSB and the charge absorption, indicating that some ultrafast HT has already occurred within the time resolution, most probably from the intercalated PCBM molecules (as in the $1: 1$ blend). The subsequent evolution of the spectra is however very different from what was observed in the other three experiments. In particular, there is a progressive rise of the pBTTT GSB signal, as the polymer's charged state is indirectly populated by delayed HT. The process is slow, because it concerns excitons that are generated in the pure PCBM clusters, which need to diffuse towards pBTTT in the co-crystals. There is also a weak rise of the charge absorption band above $700 \mathrm{~nm}$. This rise might be partially obscured by the overlapping flat absorption of the decaying PCBM exciton. ${ }^{\mathbf{4 9} 50}$ Unlike the dynamics of the 1:1 blend, which are quite similar for both excitation wavelengths, the differences in the $1: 4$ blends are much more pronounced (Fig. 3). With $390 \mathrm{~nm}$ excitation, there is a multiphasic increase of the GSB at $605 \mathrm{~nm}$, which continues 
beyond the 1 ns time window. An increase is also seen in the charge absorption $(720 \mathrm{~nm}, 850 \mathrm{~nm})$, but it competes at long time delays with a weak decay due to $\mathrm{gCR}$, as was observed upon $540 \mathrm{~nm}$ excitation.

For the global analysis, we needed three largely different time constants (690 fs, $20.6 \mathrm{ps}$ and $485 \mathrm{ps}$ ) to reproduce the dynamics due to delayed HT with $390 \mathrm{~nm}$ excitation of the $1: 4$ blend. This is most likely a crystallisation of a much broader distribution of time constants related to the process. ${ }^{39}$ The corresponding amplitude spectra are characterized by a structured positive band mirroring the GSB signal (inset of Fig. 2C). The shift of the fastest (690 fs) component suggests entanglement with the red shift of the GSB clearly seen in the TA spectra (probably Stark effect). The strongly multiphasic dynamics of the delayed HT originates from a variety of distances of the excitons generated in the PCBM clusters from the co-crystals. It is likely that not all excitons reach pBTTT during their lifetime (as previously suggested by incomplete quenching of the fullerene emission), especially if the PCBM domains are larger than the $5 \mathrm{~nm}$ exciton diffusion length. ${ }^{26,27}$ Although we have discussed HT as splitting of a PCBM exciton in the presence of pBTTT, we cannot exclude the alternative scenario of hole injection into the polymer following spontaneous charge dissociation within the PCBM clusters and charge migration (this would lead to similar evolution of the TA spectra). The process has been demonstrated, ${ }^{51,52}$ but is probably slower than what we observe here and therefore has a minor influence on our results. ${ }^{53}$

In summary, we have shown that charge separation in the completely intercalated $1: 1$ blend predominantly occurs on the ultrafast ( $\sim 100 \mathrm{fs}$ ) time scale, no matter whether the pBTTT or PCBM is initially excited. Thus, dissociation of the neutral exciton by either electron or hole transfer is extremely fast when the donor and acceptor are in intimate contact. The dynamics following the initial CS step by ET or HT are quite similar, pointing to a comparable evolution of the charges. Most of them undergo geminate recombination ( $\mathrm{gCR}$ ), leading to the low corresponding solar cell efficiency. When pBTTT is selectively excited in the 1 : 4 blend, ET is still ultrafast, since a noticeable fraction of fullerene remains intercalated with the polymer in a molecularly mixed phase. Nevertheless, most of the charges survive beyond the investigated 1 ns time window, supporting the idea that the presence of pure PCBM aggregates prevents gCR by providing a driving force for spatial separation of charges formed in the co-crystals. ${ }^{26}$ The situation becomes very different when PCBM is excited in the $1: 4$ blend. The CS dynamics is now dominated by strongly multiphasic HT ranging from the ultrafast to slow ( $\sim 500 \mathrm{ps})$ time scale. The slow components are due to PCBM excitons within the fullerene clusters that need to diffuse over various distances to a polymer prior to HT. Some of them might never undergo CS during their lifetime.

\section{Part II: charge generation in PBDTTPD:PCBM}

The other investigated blend, PBDTTPD:PCBM, gives us access to a third kind of microstructure containing also relatively phase-pure polymer domains, so that we can investigate whether their presence delays the ET process. For the P3 sidechain derivative of PBDTTPD (Fig. 1B), an X-ray diffraction study of the optimized fullerene blend has demonstrated the presence of pure polymer aggregates (more disordered than crystalline), pure PCBM clusters and regions where the fullerenes mix at the molecular level with amorphous PBDTTPD. ${ }^{24}$ This is schematically shown in Fig. 1B. The PCBM clusters appear at a fullerene concentration above $20 \%$ by weight. PCBM below this concentration mixes with a limited amount of PBDTTPD, since it was shown that the intermixed regions must be PCBM-rich in order to provide the necessary percolated pathways for electron transport. ${ }^{24}$ According to this reasoning, most PBDTTPD is found in the pure polymer aggregates.

In addition to studying excitation wavelength dependent ET and HT processes in the P3:PCBM blend, we have investigated the effect of backbone packing in the pure polymer aggregates on the ET dynamics. The PBDTTPD polymer bears alkyl sidechains on both the TBD (thienopyrroledione) and BDT (benzodithieophene) units, which are necessary for solubility (Fig. 1B). It has been shown that the length and bulkiness of the sidechains affect the solid-state microstructure, in particular the packing ( $\pi$-stacking) of the polymer backbones, the backbone orientation with respect to the substrate, and the long-range ordering. ${ }^{2,35}$ The three derivatives (P1-P3) investigated here bear different chains on the TPD unit. The highest ordering, short $\pi$-stacking distance and face-on orientation with the linear sidechain of P3 leads to the best solar cell efficiency in the PCBM blend (6.4\%), compared to only $3.9 \%$ and $2.8 \%$ for P2 and P1, respectively. ${ }^{35}$ The efficiency can be further enhanced by shortening the TPD alkyl chain by one carbon (up to $8.5 \%$ ). ${ }^{2}$ However, replacing the branched chains on the BDT with linear ones strongly reduces the photocurrent yield because the faceon orientation is lost. ${ }^{2}$

The absorption spectra of the PBDTTPD (P3, $14 \mathrm{kDa})$ and PBDTTPB:PCBM (P3, $14 \mathrm{kDa}, 1: 2)$ films used in the wavelengthdependent study are shown in Fig. 1C (second panel). Unlike with PBTTT, the broad structured absorption band of PBDTTPD (400-750 nm) does not significantly change between the neat and blended sample, pointing to similar aggregation of the polymer, which is in agreement with X-ray studies. ${ }^{35}$ The orbital energy levels of the side-chain derivatives are similar, given the same conjugated backbone. ${ }^{35}$ However, different polymer packing leads to shifts in the thin film absorption spectra, particularly in the first (low energy) vibronic peak. The spectra are shown for the PCBM blends of P1-P3 $(1: 1.5)$ in the third panel of Fig. 1C. The absorption band of P3 is the broadest and most red shifted, followed by weakly shifted P2 and most blue shifted P1. The red shift in P3 favours harvesting of the solar spectrum and has been attributed to good polymer ordering leading also to backbone planarization in the solid state (increased conjugation length and delocalization). ${ }^{35}$ At higher molecular weight, the red side of the first vibronic peak is generally also broadened, as seen for the $6 \mathrm{kDa}$ and $28 \mathrm{kDa}$ P1 samples, and the $27 \mathrm{kDa}$ and $36 \mathrm{kDa}$ P3 samples. Optimal solar cell efficiency for P3 is obtained with the highest molecular weight. ${ }^{34,35}$

In Fig. 4A and B, the TA spectra of the P3:PCBM blend exited at $390 \mathrm{~nm}$ (mainly PCBM absorption) and at $530 \mathrm{~nm}$ (mainly 
PBDTTPD absorption) are shown for selected time delays. They were recorded by very carefully keeping the number of absorbed photons constant. Conveniently, the signature of positively charged PBDTTPD is highly characteristic, with a flat absorption in the 660-1000 nm region and a pronounced peak at 880 $\mathrm{nm}$ (shown by TA and PIA spectroscopy as well as absorption of the oxidized polymer). ${ }^{12,54}$ At the longest timedelay (1000 ps, blue curve), the TA spectra of PBDTTPD:PCBM are similar for both excitation wavelengths. They are dominated by the polymer's GSB (460-660 nm) and the characteristic charge absorption (880 $\mathrm{nm}$ peak), indicating that charges have been efficiently generated by ET or HT. However, in contrast to the pBTTT:PCBM samples, where the spectral shape hardly evolved except at ultrafast times, the early TA spectra of PBDTTPD:PCBM are clearly different from the late ones. For both excitation wavelengths, the $880 \mathrm{~nm}$ band is only weakly pronounced at $150 \mathrm{fs}$, pointing to limited prompt CS and additional delayed CS components.

Upon polymer absorption (530 nm, Fig. 4B), the signatures of the neutral PBDTTPD exciton are also visible at early time delays. They include the negative SE band on the red side of the GSB (640-750 nm, coincident with the steady-state emission), ${ }^{54}$ and the broad excited state absorption (ESA, 740-1000 nm, rising at long wavelengths), as identified by comparison to the neat PBDTTPD spectrum shown in the inset of Fig. 4B (in grey). The similar aggregation in the neat and blended polymer allows this direct comparison. In the same inset, we subtracted the scaled PBDTTPD exciton signature from the 150 fs spectrum of the blend and obtained the characteristic spectrum of charges. Thus, some ultrafast ET indeed occurs before $150 \mathrm{fs}$, but the process is not complete. In fact, the amplitude of the $880 \mathrm{~nm}$ peak at 150 fs (after subtracting the ESA contribution) is only about $25 \%$ of that at late time delays. Some prompt HT also occurs in the blend upon $390 \mathrm{~nm}$ excitation, since the polymer's GSB and charge absorption are partly present at $150 \mathrm{fs}$, in spite of predominant PCBM excitation (Fig. 4A). Prompt CS can in principle occur for excitons generated near the interface of pure domains, or for excitons generated in the intermixed phase. We have shown elsewhere that the fraction of prompt CS depends predominantly on the amount of the intermixed phase, suggesting that this is where prompt CS happens. ${ }^{49}$ Thus, the small fraction of CS occurring promptly is consistent with the small amount of intimately mixed phase present in the PBDTTPD:PCBM blend. It is possible that the pure domains are not in direct contact, but separated by the mixed phase, but evidencing this goes beyond the scope of the present manuscript.

Most ET and HT is delayed and not prompt in PBDTTPD:PCBM, due to the presence of the pure polymer and fullerene regions, making exciton diffusion to a CS site necessary. This can either be an interface between the pure domains (if they are in contact), or the intermixed region. At this point we cannot distinguish between the two. In Fig. 4A (390 nm excitation), there is a rise of the GSB and $880 \mathrm{~nm}$ peak, mainly between the 10 ps and 1000 ps spectra. Similarly as with the $1: 4$ blend of pBTTT:PCBM, this is characteristic of slow HT
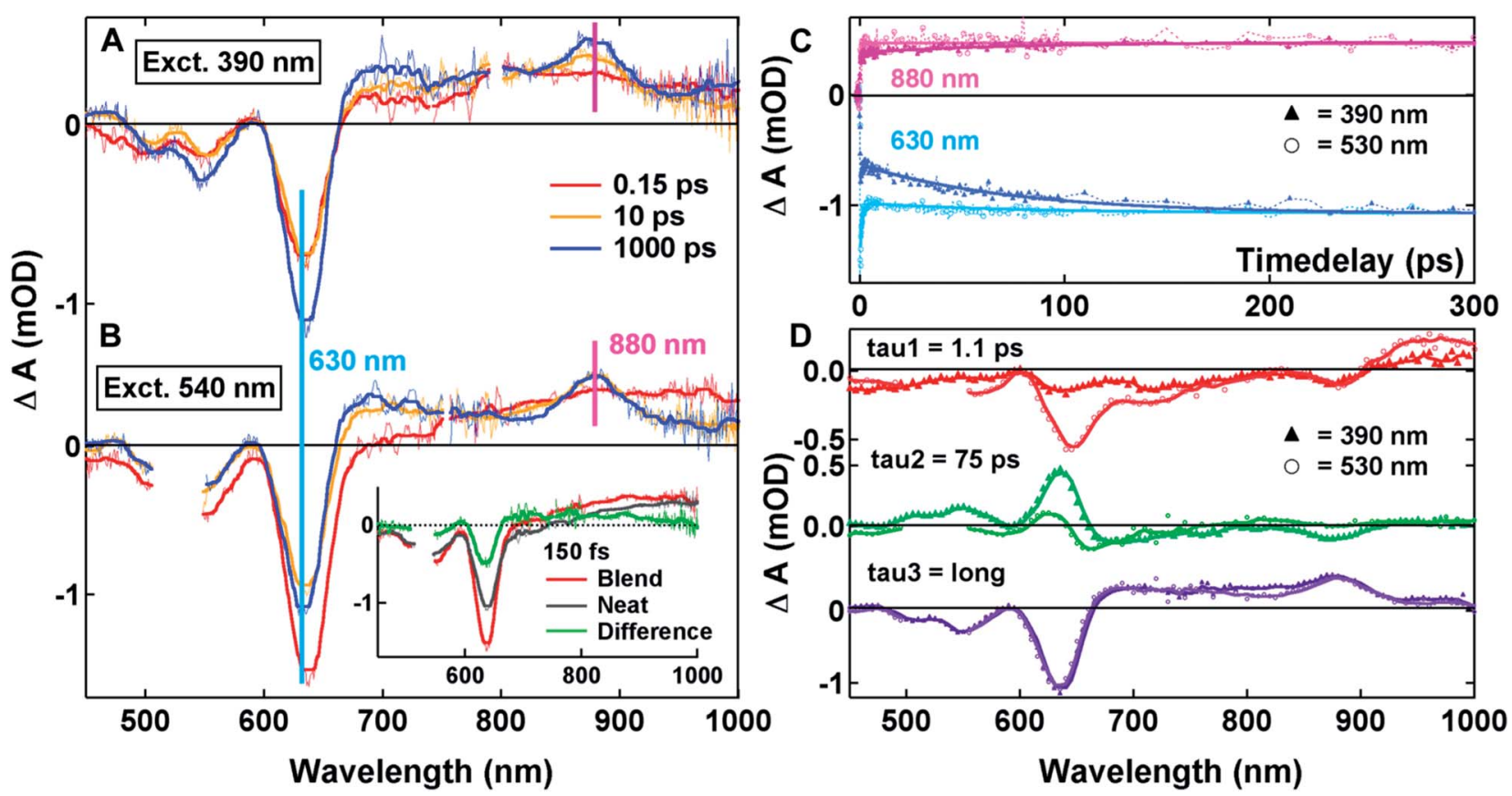

Fig. 4 (A and B) TA spectra of the P3 derivative of PBDTTPD (14 kDa) blended with PCBM (1:2) recorded at selected time delays following excitation at $390 \mathrm{~nm}$ and $540 \mathrm{~nm}$, respectively. Thick solid lines are smoothed and overlaid to the raw experimental data (thin lines). The inset shows an estimation of the spectrum of promptly generated charges at $150 \mathrm{fs}$, obtained by subtracting the scaled contribution of the neutral exciton measured in the neat PBDTTPD film. (C) TA dynamics probed at $880 \mathrm{~nm}$ (charge absorption) and $630 \mathrm{~nm}$ (GSB, SE) compared for the two excitation wavelengths (slightly darker colour was used for $390 \mathrm{~nm}$ ). Solid lines represent the best multiexponential fit. (D) Amplitude spectra associated with the time constants obtained by multiexponential global analysis of the data. 
following diffusion of PCBM excitons through pure fullerene clusters. The slow rise of the GSB $(630 \mathrm{~nm})$ and charge peak $(880 \mathrm{~nm})$ is clearly visible in the dynamics (Fig. 4C), as well as in the amplitude spectrum associated with the related $75 \mathrm{ps}$ global time constant (Fig. 4D). Again, this is an average time scale obtained by exponential analysis, most probably representative of a more multiphasic process caused by different exciton distances from a CS site. ${ }^{39}$ We note that HT in PBDTTPD:PCBM is overall faster than in the $1: 4$ pBTTT:PCBM blend, pointing to smaller fullerene clusters. Smaller PCBM domains also explain why the process is less multiphasic (we could use a single time constant), since the distribution of distances is more limited. Based on the reported exciton diffusion coefficient in $\mathrm{C}_{60}$ films of $7.5 \times 10^{-4} \mathrm{~cm}^{2} \mathrm{~s}^{-1}, 55$ we roughly estimate that the fullerene excitons diffuse by about $2.5 \mathrm{~nm}$ within $75 \mathrm{ps}$, which allows us to estimate the diameter of the PCBM clusters to $5 \mathrm{~nm}$. This is small enough to ensure complete quenching during the lifetimes of the excitons, unlike in the pBTTT sample. The analysis remains of course simplistic, because delocalization between coupled PCBM molecules is not accounted for. ${ }^{\mathbf{5 2}}$

In Fig. 4B (polymer excitation at $530 \mathrm{~nm}$ ), the spectral changes are clearly different from the ones caused by slow HT. They are dominated by a fast (between 0.15 ps and $10 \mathrm{ps}$ ) disappearance of the polymer's exciton features (SE, ESA) and a rise of the $880 \mathrm{~nm}$ charge band, as expected for delayed ET from pure PBDTTPD domains. There is a predominant fast component in the corresponding TA dynamics in Fig. 4C probed at $630 \mathrm{~nm}$ (decay of SE) and at $880 \mathrm{~nm}$ (rise of charges). This was globally analysed with a time constant of $1.1 \mathrm{ps}$. The amplitude spectrum shown in Fig. 4D confirms the concomitant decay of the negative SE band, rise of charge absorption and decay of positive ESA at long wavelengths. We note that the fast component in the dynamics is weakly present also for $390 \mathrm{~nm}$ excitation, indicating delayed ET due to residual absorption of the polymer (about 30\%) at this wavelength. It was globally analysed using the same 1.1 ps time constant and yields an amplitude spectrum similar in shape to the one associated with ET upon $530 \mathrm{~nm}$ excitation (but smaller in size since less PBDTTPD is excited, Fig. 4D). Analogously, some PCBM (about $20 \%$ ) is excited at $530 \mathrm{~nm}$, explaining the observed small rise of the polymer GSB by slow HT (between 10 ps and 1000 ps in Fig. 4B), in addition to the fast dynamics caused by ET. The amplitude spectrum associated with the corresponding 75 ps time constant has a slightly different shape than upon $390 \mathrm{~nm}$ excitation (for example negative indent around $660 \mathrm{~nm}$ ). We ascribe this to a weak overlapping contribution of gCR and more delayed ET, as discussed in detail elsewhere. ${ }^{49}$

Apart from the rather small contribution $(<20 \%)$ of $\mathrm{gCR}$ masked by the rise of the signals due to the slow HT, ${ }^{\mathbf{4 9 , 5 4}}$ we do not see any evidence for significant decay of the GSB and charges related to recombination, in agreement with high solar cell efficiency of PBDTTPD:PCBM. This shows that excitons initially formed in either the intermixed regions or pure domains are successfully converted to free charges, after their dissociation by ET or HT. In further support of the equally efficient HT and ET pathways, we note the same signal amplitude of the $1 \mathrm{~ns}$ spectra recorded at both excitation wavelengths with a similar flux of absorbed photons (Fig. 4A and B). This also reflects in the converging dynamics at long time delays and the overlapping amplitude spectra related to the long-lived signal (Fig. 4C and D). The excellent efficiency of CS in PBDTTPD:PCBM is also confirmed elsewhere by $99.7 \%$ emission quenching. ${ }^{12}$ Free charges are therefore generated in quasi quantitative yield by both the ET and HT pathways, even if the driving force for HT and ET is different and populates the CT state with more or less excess energy. ${ }^{11,12}$ This is evidence that charges formed in the PBDTTPD:PCBM blend (at domain interfaces or in the intermixed phase) are particularly well spatially separated, possibly thanks to the driving force provided by the pure regions, or to the delocalization in the polymer (see below).

Another factor leading to high solar cell efficiency with PBDTTPD is the ability of the polymer to undergo very fast and efficient ET in spite of necessary exciton diffusion out of predominant pure domains. Even if delayed, a time scale of 1.1 ps for ET preceded by exciton migration through PBDTTPD aggregates is still extremely fast. It is not consistent with typical exciton diffusion coefficients of $2-3 \times 10^{-3} \mathrm{~cm}^{2} \mathrm{~s}^{-1}$ reported for conjugated polymers, ${ }^{\mathbf{5 6}, 57}$ according to which point-like excitons cover only $0.5 \mathrm{~nm}$ within $1 \mathrm{ps}$. We therefore suggest that the pure PBDTTPD domains are fairly small (a few nanometers diameter), that exciton migration out of those regions occurs essentially intramolecularly along the same polymer chain, and that it is helped by delocalization. In support of this, we have undertaken elsewhere a very detailed analysis of the distance dependence of the prompt and delayed ET and its interplay with relaxation and delocalization. ${ }^{49}$ Depending on the inter- and intra-chain coupling between chromophore units, exciton hopping (typically by excitation energy transfer between segments formed by conjugation breaks) can either be faster within the chain or between chains., ${ }^{\mathbf{9 5 8 - 6 0}}$ For the intramolecular case, which we stipulate for PBDTTPD, delocalization of the exciton allows it to sample a greater spatial extent of the backbone, so that it requires less transfer steps to cover a certain distance. Gas-phase calculations and solution spectroscopy have indeed shown that PBDTTPD is particularly planar in both the ground and excited state, favouring exciton delocalization. ${ }^{\mathbf{6 1 6 2}}$ This delocalization is possibly further enhanced at ultrashort times. ${ }^{8}$ Given the good ordering in the polymer domains of P3:PCBM, the backbone planarization is maintained in the solid state, ${ }^{35}$ possibly contributing to fast intrachain exciton diffusion.

To support the intrachain exciton diffusion mechanism, we will now examine the effect of polymer packing, chain length and backbone planarity on the ET dynamics, by investigating different side-chain derivatives and molecular weights of PBDTTPD. Intrachain exciton hopping to a CS site implies that changing the packing between the polymer chains in the pure aggregates should have little effect. We verified this by comparing P3:PCBM to the P2 and P1 blends, in which the polymer is less ordered, but which should have a similar chain extension given the same conjugated backbone and comparable molecular weight $(28 \mathrm{kDa})$. The evolution of the TA spectra for the three samples upon $530 \mathrm{~nm}$ excitation is very similar 
(Fig. 5A), in spite of the large variation in solar cell efficiency. ${ }^{35}$ The promptly appearing charge signature is always weak (thus the fraction of the intermixed phase in a three-phase microstructure remains low for P1 and P2), while there is always a significant long-lived component corresponding to efficiently generated charges. In-between, the typical features of delayed ET are seen (decay of the SE to the left of the GSB, rise of the charge absorption band, decay of the ESA above $920 \mathrm{~nm}$ ). We note that while the GSB is blue-shifted in P1 (as the steady-state absorption), the charge peak is a little broader but still at 880 $\mathrm{nm}$. For the three side-chain derivatives, a fast component is clearly visible in the TA dynamics at selected probe wavelengths (Fig. 5B) and could be globally analysed using the 1.1 ps time constant and yielding an amplitude spectrum (not shown) similar to the one in Fig. 4D, confirming assignment to delayed ET. The presence of the fast ET component in spite of the reduced $\pi$-stacking order in the $\mathrm{P} 2$ and $\mathrm{P} 1$ blends shows that the related fast exciton diffusion is essentially intrachain.

Minor, but reproducible differences between the three derivatives are still visible. In particular for P1:PCBM $(28 \mathrm{kDa})$, spectral changes related to quenching of the SE around $650 \mathrm{~nm}$ at longer time delays are more pronounced (1-200 ps, Fig. 5A). For this sample, the slower decay at $619 \mathrm{~nm}$ (SE and GSB) and at $647 \mathrm{~nm}$ (mainly SE) is also visible in the TA dynamics, in addition to the fast $1.1 \mathrm{ps}$ component (red curves in Fig. 5B, normalized at long times). The effect is even enhanced if the molecular weight of P1 is reduced to only $6 \mathrm{kDa}$ (orange dynamics). Now, slower quenching of the ESA is also seen at $950 \mathrm{~nm}$. We obtained a global $62 \mathrm{ps}$ time constant related to the slower process in P1:PCBM. The blue shifted absorption band and previous X-ray study of P1:PCBM suggest that the polymer backbone is less planar in more disordered aggregates. ${ }^{35}$ Enhanced conjugation breaks can therefore reduce the delocalization of the exciton and slow down its migration along the backbone, leading to the slower ET component. Delocalization is further reduced in the very short $6 \mathrm{KDa}$ chains. It can be observed in Fig. 5A that the GSB signal is generally smaller for $\mathrm{P} 1$ and P2, especially for the $6 \mathrm{kDa}$ sample (in spite of similar steady-state absorption and scaling to account for the absorbed photon flux). The GSB cross-section is reduced because the less delocalized excitons span less chromophores along the conjugated backbone. An additional reason for slower ET in the $6 \mathrm{kDa}$ sample is that the chains might not be long enough to span the entire pure domains, so that interchain exciton hopping becomes necessary.

In order to confirm that the 62 ps component is related to slow ET for some of the excitons in the P1:PCBM blends, the corresponding amplitude spectra are compared to the one associated with the 75 ps time constant in $27 \mathrm{kDa}$ P3:PCBM (Fig. 5C, same colour coding as the dynamics). The shape of the latter $75 \mathrm{ps}$ spectrum is similar to the corresponding one in Fig. 4C (both with $530 \mathrm{~nm}$ excitation), although a different polymer batch of P3 and slightly lower fullerene concentration was used. It can likewise be assigned to slow HT from remaining PCBM excitation, mixed with a weak contribution of slow ET (possibly for excitons trapped at conjugation breaks or which need to jump chains) and/or with weak gCR. We obtained a similar amplitude spectrum and 75 ps time constant for the
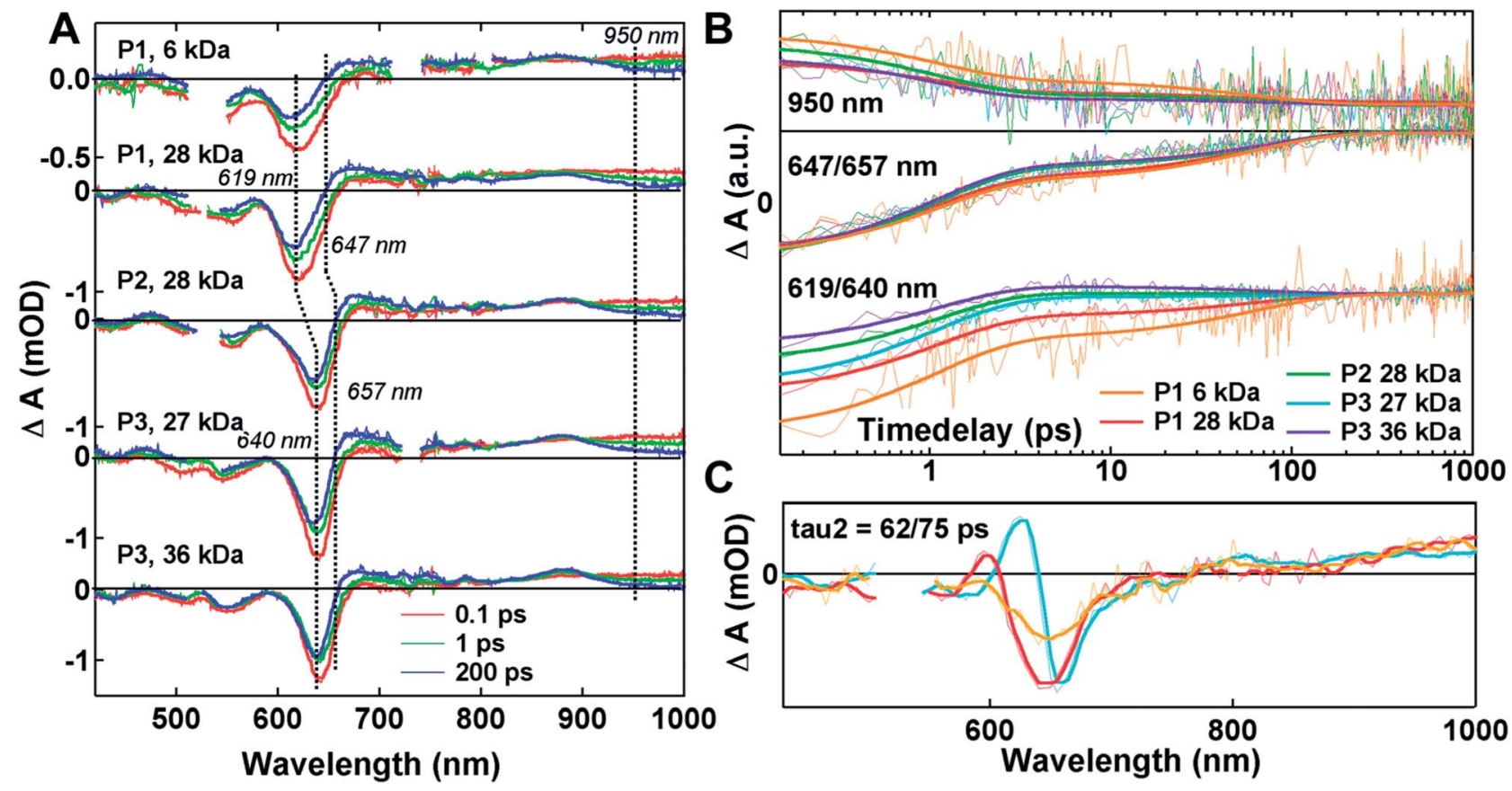

Fig. 5 (A) TA spectra at selected time delays for different side-chain derivatives and molecular weights of PBDTTPD (P1, P2 and P3) blended with PCBM (1: 1.5) and recorded following $530 \mathrm{~nm}$ excitation. Spectra were slightly scaled to account for the number of absorbed photons, the thicker lines are smoothed as guide to the eye. (B) Corresponding dynamics at selected probe wavelengths, normalized at long time delays and shown on a logarithmic scale. Solid lines represent the best multiexponential fit. (C) Amplitude spectra associated with the intermediate time constant (62 ps or 75 ps) for 27 kDa P3, 28 kDa P1 and 6 kDa P1. 
blends of P2 (28 kDa) and of a P3 batch with very high molecular weight $(36 \mathrm{kDa})$, not shown. However, the shape of the 62 ps amplitude spectrum for P1:PCBM shows much more pronounced decay of the polymer's SE (negative signal around $650 \mathrm{~nm}$ ), which completely masks the positive rise of the GSB due to slow HT for the $6 \mathrm{kDa}$ sample (red and orange curves in Fig. 5C). Here, the amplitude spectra resemble very much the one associated with the fast 1.1 ps ET process ( $\tau_{1}$ in Fig. 4D), revealing decay of the SE and ESA together with weak rise of the charge peak. The indent due to the rise of charge absorption at $880 \mathrm{~nm}$ is actually not very pronounced, which might indicate that some of the excitons decay to the ground state because they never reach a CS site. We have thus established that the $62 \mathrm{ps}$ component in the P1 blends is related to an enhanced slow ET component (and possibly some exciton loss), present due to reduced delocalization and chain length.

Finally, the TA data related to the $36 \mathrm{kDa}$ P3 polymer, which gives the highest solar cell efficiency, show indeed some positive characteristics. For example, the recovery of the GSB below $600 \mathrm{~nm}$ is less pronounced than in the other samples (Fig. 5A), pointing to minimal recombination. Also, the appearance of charges due to ET seems to be fastest according to the dynamics in Fig. 5B (mainly within $1.1 \mathrm{ps}$, less slow component). However, the differences compared to the other samples are very small. All the PBDTTPD blends show in fact quite efficient generation of long-lived charges. Therefore, the important variation in solar cell efficiency mostly stems from charge transport effects occurring at much slower time scales than the ones investigated here. Indeed, it has been shown that good short-range packing of polymer chains as well as connectivity between aggregates provided by high molecular weight chains are most important for efficient transport. ${ }^{63}$ From what we have discussed, those conditions are fulfilled best in the $36 \mathrm{kDa}$ batch of P3, which additionally has a favourable face-on orientation of the backbones with respect to the substrate. ${ }^{35}$

In summary, we have shown that the three-phase microstructure in P3-PBDTTPD:PCBM leads to three distinct charge separation pathways: prompt ET and HT occurs in the intermixed phase $(<100 \mathrm{fs})$, slow HT occurs from the PCBM clusters ( $\sim 75 \mathrm{ps}$ ), and delayed (but still very fast) ET occurs from the PBDTTPD aggregates ( 1.1 ps). The yield of CS is quantitative in all three cases and we did not notice very significant geminate recombination. This shows that both ET and HT very efficiently yield charges, that the pure regions are sufficiently small (and the polymer excitons sufficiently delocalized) to allow complete exciton quenching, and that carriers generated in the intermixed phase can be spatially separated, possibly thanks to the driving force provided by the pure domains. Our results are in excellent agreement with the high reported internal quantum efficiency (IQE) of 0.9 , which is flat across the visible spectrum. ${ }^{24}$ Only minor changes to the ET dynamics occur for different molecular weights of P3 and for the P2 and P1 side-chain derivatives, pointing to a similar three-phase microstructure in all cases. The small effect of polymer packing on the fast ET from pure PBDTTPD regions confirms that exciton diffusion out of the pure domains essentially occurs within the same chain, helped by delocalization. Indeed, a slower ET component (60-70 ps) is enhanced in the P1:PCBM blend, which has a less planar backbone (less delocalization), and for short chains (low molecular weight). The main reduction of solar cell efficiency for bulky side-chains and low molecular weight is however not caused by poor charge generation, but probably by poor transport.

\section{Conclusion}

We have investigated the charge generation dynamics upon selective excitation of either the fullerene or the polymer in different bulk heterojunction blends with well-characterized microstructure. We found a strong correlation of the electron and hole transfer rates, as well as of the subsequent generation of free charges, with the presence of phase-pure regions and intermixed domains. Our work brings new insights into important questions related to charge generation in organic solar cells and emphasizes the role played by the microstructure. It also identifies ultrafast spectroscopy as a potential tool to predict the microstructure of blends when it is not previously known.

\section{Acknowledgements}

N.B. thanks the Swiss National Science Foundation for funding through the Ambizione Fellowship PZ00P2_136853, as well as Prof. Kevin Sivula and his group for assistance regarding sample preparation.

\section{Notes and references}

1 M. A. Green, K. Emery, Y. Hishikawa, W. Warta and E. D. Dunlop, Prog. Photovolt: Res. Appl., 2013, 21, 827.

2 C. Cabanetos, L. A. El, J. A. Bartelt, J. D. Douglas, W. R. Mateker, J. M. J. Fréchet, M. D. McGehee and P. M. Beaujuge, J. Am. Chem. Soc., 2013, 135, 4656.

3 M. Zhang, Y. Gu, X. Guo, F. Liu, S. Zhang, L. Huo, T. P. Russell and J. Hou, Adv. Mater., 2013, 25, 4944.

4 Z. C. He, C. M. Zhong, S. J. Su, M. Xu, H. B. Wu and Y. Cao, Nat. Photonics, 2012, 6, 591.

5 A. J. Heeger, N. S. Sariciftci and E. B. Namdas, Semiconducting and Metallic Polymers, Oxford University Press Oxford, UK, 2010.

6 B. C. Thompson and J. M. J. Fréchet, Angew. Chem., Int. Ed., 2008, 47, 58.

7 G. Yu, J. Gao, J. C. Hummelen, F. Wudl and A. J. Heeger, Science, 1995, 270, 1789.

8 N. Banerji, J. Mater. Chem. C, 2013, 1, 3052.

9 N. Banerji, S. Cowan, M. Leclerc, E. Vauthey and A. J. Heeger, J. Am. Chem. Soc., 2010, 132, 17459.

10 C. J. Brabec, G. Zerza, G. Cerullo, S. De Silvestri, S. Luzzati, J. C. Hummelen and S. Sariciftci, Chem. Phys. Lett., 2001, 340, 232.

11 A. A. Bakulin, S. D. Dimitrov, A. Rao, P. C. Y. Chow, C. B. Nielsen, B. C. Schroeder, I. McCulloch, H. J. Bakker, J. R. Durrant and R. H. Friend, J. Phys. Chem. Lett., 2012, 4, 209. 12 E. T. Hoke, K. Vandewal, J. A. Bartelt, W. R. Mateker, J. D. Douglas, R. Noriega, K. R. Graham, J. M. J. Fréchet, 
A. Salleo and M. D. McGehee, Adv. Energy Mater., 2013, 3, 220.

13 Y. Fang, A. K. Pandey, A. M. Nardes, N. Kopidakis, P. L. Burn and P. Meredith, Adv. Energy Mater., 2013, 3, 54.

14 S. D. Dimitrov, C. B. Nielsen, S. Shoaee, T. P. Shakya, J. Du, I. McCulloch and J. R. Durrant, J. Phys. Chem. Lett., 2012, 3, 140.

15 I. A. Howard, R. Mauer, M. Meister and F. Laquai, J. Am. Chem. Soc., 2010, 132, 14866.

16 A. A. Bakulin, J. C. Hummelen, M. S. Pshenichnikov and P. H. M. van Loosdrecht, Adv. Funct. Mater., 2010, 20, 1653.

17 S. Cook, R. Katoh and A. Furube, J. Phys. Chem. C, 2009, 113, 2547.

18 M. M. Wienk, J. M. Kroon, W. J. H. Verhees, J. Knol, J. C. Hummelen, P. A. van Hal and R. A. J. Janssen, Angew. Chem., Int. Ed., 2003, 42, 3371.

19 N. C. Miller, E. Cho, R. Gysel, C. Risko, V. Coropceanu, C. E. Miller, S. Sweetnam, A. Sellinger, M. Heeney, I. McCulloch, J.-L. Bredas, M. F. Toney and M. D. McGehee, Adv. Energy Mater., 2012, 2, 1208.

20 P. M. Beaujuge and J. M. J. Fréchet, J. Am. Chem. Soc., 2011, 133, 20009.

21 B. A. Collins, J. R. Tumbleston and H. Ade, J. Phys. Chem. Lett. , 2011, 2, 3135.

22 A. C. Mayer, M. F. Toney, S. R. Scully, J. Rivnay, C. J. Brabec, M. Scharber, M. Koppe, M. Heeney, I. McCulloch and M. D. McGehee, Adv. Funct. Mater., 2009, 19, 1173.

23 N. C. Cates, R. Gysel, Z. Beiley, C. E. Miller, M. F. Toney, M. Heeney, I. McCulloch and M. D. McGehee, Nano Lett., 2009, 9, 4153.

24 J. A. Bartelt, Z. M. Beiley, E. T. Hoke, W. R. Mateker, J. D. Douglas, B. A. Collins, J. R. Tumbleston, K. R. Graham, A. Amassian, H. Ade, J. M. J. Fréchet, M. F. Toney and M. D. McGehee, Adv. Energy Mater., 2013, 3, 364.

25 P. Westacott, J. R. Tumbleston, S. Shoaee, S. Fearn, J. H. Bannock, J. B. Gilchrist, S. Heutz, M. J. de, M. Heeney, H. Ade, J. Durrant, D. S. McPhail and N. Stingelin, Energy Environ. Sci., 2013, 6, 2756.

26 F. C. Jamieson, E. B. Domingo, T. McCarthy-Ward, M. Heeney, N. Stingelin and J. R. Durrant, Chem. Sci., 2012, 3, 485.

27 D. R. Kozub, K. Vakhshouri, S. V. Kesava, C. Wang, A. Hexemer and E. D. Gomez, Chem. Commun., 2012, 48, 5859.

28 W. L. Rance, A. J. Ferguson, T. McCarthy-Ward, M. Heeney, D. S. Ginley, D. C. Olson, G. Rumbles and N. Kopidakis, ACS Nano, 2011, 5, 5635.

29 T. J. Savenije, W. J. Grzegorczyk, M. Heeney, S. Tierney, I. McCulloch and L. D. A. Siebbeles, J. Phys. Chem. C, 2010, 114, 15116.

30 I.-W. Hwang, J. Y. Kim, S. Cho, J. Yuen, N. Coates, K. Lee, M. Heeney, I. McCulloch, D. Moses and A. J. Heeger, J. Phys. Chem. C, 2008, 112, 7853.

31 M. Baklar, P. H. Wobkenberg, D. Sparrowe, M. Goncalves, I. McCulloch, M. Heeney, T. Anthopoulos and N. Stingelin, J. Mater. Chem., 2010, 20, 1927.

32 B. H. Hamadani, D. J. Gundlach, I. McCulloch and M. Heeney, Appl. Phys. Lett., 2007, 91, 243512.
33 I. McCulloch, M. Heeney, C. Bailey, K. Genevicius, I. MacDonald, M. Shkunov, D. Sparrowe, S. Tierney, R. Wagner, W. M. Zhang, M. L. Chabinyc, R. J. Kline, M. D. McGehee and M. F. Toney, Nat. Mater., 2006, 5, 328.

34 B. R. Aich, J. Lu, S. Beaupre, M. Leclerc and Y. Tao, Org. Electron., 2012, 13, 1736.

35 C. Piliego, T. W. Holcombe, J. D. Douglas, C. H. Woo, P. M. Beaujuge and J. M. J. Fréchet, J. Am. Chem. Soc., 2010, 132, 7595.

36 Y. P. Zou, A. Najari, P. Berrouard, S. Beaupre, B. R. Aich, Y. Tao and M. Leclerc, J. Am. Chem. Soc., 2010, 132, 5330.

37 W. R. Mateker, J. D. Douglas, C. Cabanetos, I. T. SachsQuintana, J. A. Bartelt, E. T. Hoke, L. A. El, P. M. Beaujuge, J. M. J. Fréchet and M. D. McGehee, Energy Environ. Sci., 2013, 6, 2529.

38 I. H. M. van Stokkum, D. S. Larsen and R. van Grondelle, Biochim. Biophys. Acta, Bioenerg., 2004, 1657, 82.

39 A. Siemiarczuk, B. D. Wagner and W. R. Ware, J. Phys. Chem., 1990, 94, 1661.

40 F. Dou, E. Buchaca-Domingo, M. Sakowicz, X. Zhang, N. Stingelin and C. Silva, Proceeding of SPIE: Physical Chemistry of Interfaces and Nanomaterials XII, 2013, vol. 8811, p. 1J.

41 M. J. Lee, Z. Y. Chen, R. di Pietro, M. Heeney and H. Sirringhaus, Chem. Mater., 2013, 25, 2075.

42 L. G. Kaake, J. J. Jasieniak, R. C. Bakus, G. C. Welch, D. Moses, G. C. Bazan and A. J. Heeger, J. Am. Chem. Soc., 2012, 134, 19828.

43 G. Grancini, M. Maiuri, D. Fazzi, A. Petrozza, H. J. Egelhaaf, D. Brida, G. Cerullo and G. Lanzani, Nat. Mater., 2013, 12, 29.

44 F. Etzold, I. A. Howard, R. Mauer, M. Meister, T.-D. Kim, K.-S. Lee, N. S. Baek and F. Laquai, J. Am. Chem. Soc., 2011, 133, 9469.

45 V. Gulbinas, Y. Zaushitsyn, H. Bassler, A. Yartsev and V. Sundstrom, Phys. Rev. B: Condens. Matter Mater. Phys., 2004, 70, 035215.

46 K. Vandewal, S. Albrecht, E. T. Hoke, K. R. Graham, J. Widmer, J. D. Douglas, M. Schubert, W. R. Mateker, J. T. Bloking, G. F. Burkhard, A. Sellinger, J. M. J. Fréchet, A. Amassian, M. K. Riede, M. D. McGehee, D. Neher and A. Salleo, Nat. Mater., 2014, 13, 63.

47 S. D. Dimitrov, A. A. Bakulin, C. B. Nielsen, B. C. Schroeder, J. Du, H. Bronstein, I. McCulloch, R. H. Friend and J. R. Durrant, J. Am. Chem. Soc., 2012, 134, 18189.

48 A. A. Bakulin, A. Rao, V. G. Pavelyev, P. H. M. van Loosdrecht, M. S. Pshenichnikov, D. Niedzialek, J. Cornil, D. Beljonne and R. H. Friend, Science, 2012, 335, 1340.

49 A. A. Paraecattil and N. Banerji, J. Am. Chem. Soc., 2014, 136, 1472.

50 N. Banerji, M. F. Wang, J. Fan, E. S. Chesnut, F. Wudl and J. E. Moser, J. Mater. Chem., 2012, 22, 13286.

51 G. F. Burkhard, E. T. Hoke, Z. M. Beiley and M. D. McGehee, J. Phys. Chem. C, 2012, 116, 26674.

52 S. Cook, H. Ohkita, Y. Kim, J. J. Benson-Smith, D. D. C. Bradley and J. R. Durrant, Chem. Phys. Lett., 2007, 445, 276.

53 L. W. Barbour, M. Hegadorn and J. B. Asbury, J. Am. Chem. Soc., 2007, 129, 15884. 
54 A. A. Paraecattil, S. Beaupré, M. Leclerc, J.-E. Moser and N. Banerji, J. Phys. Chem. Lett., 2012, 3, 2952.

55 M. C. Fravventura, J. Hwang, J. W. A. Suijkerbuijk, P. Erk, L. D. A. Siebbeles and T. J. Savenije, J. Phys. Chem. Lett., 2012, 3, 2367.

56 P. E. Shaw, A. Ruseckas and I. D. W. Samuel, Adv. Mater., 2008, 20, 3516.

57 A. J. Lewis, A. Ruseckas, O. P. M. Gaudin, G. R. Webster, P. L. Burn and I. D. W. Samuel, Org. Electron., 2006, 7, 452.

58 D. E. Markov and P. W. M. Blom, Phys. Rev. B: Condens. Matter Mater. Phys., 2006, 74, 085206.
59 T. Q. Nguyen, J. J. Wu, V. Doan, B. J. Schwartz and S. H. Tolbert, Science, 2000, 288, 652.

60 A. Watanabe, T. Kodaira and O. Ito, Chem. Phys. Lett., 1997, 273, 227.

61 C. Risko, M. D. McGehee and J. L. Bredas, Chem. Sci., 2011, 2, 1200.

62 I. Hwang, S. Beaupre, M. Leclerc and G. D. Scholes, Chem. Sci., 2012, 3, 2270.

63 R. Noriega, J. Rivnay, K. Vandewal, F. P. V. Koch, N. Stingelin, P. Smith, M. F. Toney and A. Salleo, Nat. Mater., 2013, 12, 1038. 